\title{
The future of genomic endocrinology
}

\section{MehulT. Dattani* and Juan-Pedro Martinez-Barbera*}

Institute of Child Health, University College London, London, UK

*Correspondence: m.dattani@ich.ucl.ac.uk; j.martinez-barbera@ich.ucl.ac

The advent of molecular biology has already transformed the landscape in endocrinology. From the early days of molecular endocrinology, when techniques such as Southern blotting were used to detect large deletions, through the introduction of PCR and sequencing with the identification of variants in single genes, and finally to the present day when the focus is shifting to whole genome sequencing and identification of epigenetic influences on phenotypic expression, represents a quantum leap in our knowledge and understanding of the genomic processes underlying normality and disease evolution. However, with increasingly sophisticated techniques becoming available, the challenges facing genomic and molecular endocrinology will become increasingly complex. We will attempt to discuss some of these challenges, which hopefully will be featured in future issues of Frontiers in Genomic Endocrinology.

A number of novel genes have been identified in association with a variety of endocrine phenotypes over the last few years. However, although mutations in a number of genes have been described in association with disorders such as hypogonadotropic hypogonadism, congenital hypopituitarism, disorders of sex development, and congenital hyperinsulinism, these account for a minority of patients with these conditions, suggesting that many more genes remain to be identified. How will these novel genes be identified? Monogenic disorders can arise as a result of genomic microdeletions or microduplications, or due to single point mutations that lead to a functional change in the relevant protein. Such disorders may also result from altered expression of a gene, and hence altered dosage of the protein. Candidate genes may be identified by utilizing naturally occurring or transgenic mouse models, and this approach has been particularly informative in the elucidation of the genetic basis of a number of disorders. Other approaches include the identification of chromosomal rearrangements using conventional karyotyping techniques, as well as novel assays such as array comparative genomic hybridization (CGH) and single nucleotide polymorphism oligonucleotide arrays (SNP arrays). These molecular methods usually result in the identification of gross abnormalities as well as submicroscopic deletions and duplications, and eventually to the discovery of single gene defects that are associated with a particular phenotype.

However, there is no doubt that the major advances in novel gene identification will be made as a result of the sequencing of the genome of affected individuals and comparison with control data that are already available. Chip techniques allow hybridization of DNA or RNA to hundreds of thousands of probes simultaneously. Microarrays are being used for mutational analysis of human disease genes. Complete sequencing of genomes or sequencing of exons that encode proteins (exome sequencing) is now possible, and will lead to the elucidation of the etiology of a number of human diseases in the next few years. High-throughput, high-density sequencing using microarray technology potentially offers the option of obtaining rapid, accurate, and relatively inexpensive sequence of large portions of the genome. One such technique is oligohybridization sequencing, which relies on the differential hybridization of target DNA to an array of oligonucleotide probes. This technique is ideally suited to the analysis of DNA from patients with defined disorders, such as disorders of sex development and retinal disease, but suffers from a relatively high false positive rate and failure to detect insertions and deletions.

The greatest strides have been made in the area of next generation sequencing (NGS), which has made possible whole genome sequence analysis. A number of technologies are available, with more being developed. Template preparation, preparation of the sequencing reaction, the sequencing itself and the data handling and analyses are critical steps in NGS. The costs involved and the data handling skills required have currently restricted NGS to research laboratories. More targeted approaches such as exome capture, which analyses the sequence of coding exons only, or targeting specific chromosomes, such as the $\mathrm{X}$-chromosome in potentially X-linked conditions, are becoming more widely available. NGS technologies are capable of identifying novel disease genes and structural chromosomal variations. For example, the molecular basis of recessive CharcotMarie-Tooth Neuropathy was identified in a pedigree using whole genome sequencing (Lupski et al., 2010).

The major challenges will be the interpretation of vast amounts of data that will be generated using these techniques. It is quite likely that many disorders that are currently believed to be monogenic may in fact be digenic or oligogenic. For example, hypogonadotropic hypogonadism has recently been shown, in some affected patients, to be a digenic disorder (Pitteloud et al., 2007). Additionally, many disorders are inherited in a dominant fashion, with variability in clinical manifestations as a result of differences in penetrance or expressivity. The mechanisms underlying variable penetrance or expressivity remain to be established, but digenicity may certainly play a role. These phenomena may also reflect the effects of multiple genetic variations modifying disease expression, and/or interactions with environmental factors. Furthermore, the phenomenon of epigenetics may be implicated in the variability in penetrance. Gene expression can be influenced by epigenetic events, such as $\mathrm{X}$-inactivation and imprinting. Epigenetic alterations and chemical modifications of DNA or chromatin proteins influence gene transcription. The sum of all epigenetic information defines the epigenome, which, in contrast to the genome, is highly variable between cells and changes within a single cell over time. Epigenetic modifications lead to phenotypic changes without alteration of DNA sequence, and may be heritable.

Genomic imprinting leads to preferential expression of an allele depending on its parental origin. In this situation, DNA 
methylation leads to silencing, i.e., suppression of gene expression on one of the chromosomes. It is of importance in disorders in which the transmission of disease is dependent on the sex of the transmitting parent and has an important role in the expression of certain genetic disorders. The two classic examples are the PraderWilli and Angelman syndromes. Deletions in PWS occur exclusively on the paternal chromosome 15 whereas Angelman syndrome is due to deletions on the maternal chromosome 15. These two syndromes may also result from uniparental disomy, i.e., by the inheritance of either two maternal chromosomes 15 (PWS) or two paternal chromosomes (Angelman syndrome). Other imprinting disorders include pseudo- and pseudopseudohypoparathyroidism as well as Russell-Silver syndrome.

Many disorders have a complex etiology involving multiple genes (polygenic disorders), often in combination with environmental and lifestyle factors (multifactorial disorders). The major health care problems, cardiovascular disease, hypertension, diabetes, obesity, asthma, and psychiatric disorders, fall into this category but it also includes certain developmental abnormalities, such as cleft palate, congenital heart defects, and neural tube defects. Compared with single gene defects, complex disorders have a low heritability and do not fit a Mendelian pattern of inheritance. Twin studies have been particularly helpful in demonstrating the importance of genetic and environmental factors. Susceptibility genes or loci can be mapped using several methods, including linkage analyses, association studies, and affected sib-pair analyses. Current efforts aim to identify these genes by establishing correlations between SNPs or SNP haplotypes and complex disorders in large populations through Genome-wide Association studies, GWAS. The results of GWAS may, in part, depend on ethnicity and ascertainment criteria. Nevertheless, GWAS has been used in order to identify the genetic basis of a number of complex disorders. Human height is a classic, highly heritable, quantitative, polygenic trait; up to $90 \%$ of normal variation in human height within populations is due to genetic variation. However, although GWAS studies have identified a number of genetic variants associated with height, these account for only $\sim 3-4 \%$ of the variance in adult height. Similar data exist for obesity. Hence the challenge must be to identify other factors associated with obesity and short stature. Additionally could the genetic variants identified to date account for more extreme phenotypes?

It is often difficult to perform studies in humans, and so the generation of animal models may be valuable in understanding the etiology and pathogenesis of disease. A number of naturally occurring mouse models have led to the identification of corresponding candidate genes in humans, with mutations subsequently detected in human patients. More frequently, genes of interest are often deleted and lead to the generation of disease models. In general, mouse models correlate well with human disease; however species-specific defects need to be taken into account. Additionally, the transgenic models could be used to manipulate a condition, with the potential for new therapies. The advent of conditional transgenesis has led to an exponential increase in our understanding of how the mutation of a single gene impacts on a single organ. Using technology such as inducible gene expression systems, the effect of switching on or switching off a gene at a particular stage in development can be determined.

Advances in genomics will also have a major impact on therapeutics. Micro RNAs (miRNA) are small non-coding RNAs that regulate gene expression by targeting mRNAs of protein coding genes or noncoding RNA transcripts. Micro RNAs also have an important role in developmental and physiological processes and can act as tumor suppressors or oncogenes in the ontogenesis of cancers. The use of small interfering RNA (siRNA) offers promise of novel therapies in a range of conditions, such as cystic fibrosis and Type II autosomal dominant IGHD (Shariat et al., 2008). Elucidation of the genetic basis of disease also allows more direct targeting of therapy. For instance, children with permanent neonatal-onset diabetes mellitus (PNDM) due to mutations in SUR1 or KIR6.2 were previously treated with insulin but have now been shown to respond well to sulfonylureas, thereby allowing the cessation of insulin therapy (Pearson et al.,
2006). Finally, we are now entering the era of pharmacogenetics when the response of an individual to various therapeutic agents may be determined by their genotype. For example, a polymorphism in the GH receptor that results in deletion of exon 3 may be associated with an improved response to GH (Dos Santos et al., 2004). Thus the elucidation of the genetic basis of many disorders will aid their management, and permit the tailoring of therapy in individual patients.

\section{REFERENCES}

Dos Santos, C., Essioux, L., Teinturier, C., Tauber, M., Goffin, V., and Bougneres, P. (2004). A common polymorphism of the growth hormone receptor is associated with increased responsiveness to growth hormone. Nat. Genet. 36, 720-724.

Lupski, J. R., Reid, J. G., Gonzaga-Jauregui, C., Rio Deiros, D., Chen, D. C., Nazareth, L., Bainbridge, M., Dinh, H., Jing, C., Wheeler, D. A., McGuire, A. L., Zhang, F., Stankiewicz, P., Halperin, J. J., Yang, C., Gehman, C., Guo, D., Irikat, R. K., Tom, W., Fantin, N. J., Muzny, D. M., and Gibbs, R.A. (2010). Whole-genome sequencing in a patient with Charcot-Marie-Tooth neuropathy. N. Engl. J. Med. 362, 1181-1191.

Pearson, E. R., Flechtner, I., Njølstad, P. R., Malecki, M. T., Flanagan, S. E., Larkin, B., Ashcroft, F. M., Klimes, I., Codner, E., Iotova, V., Slingerland, A. S., Shield, J., Robert, J. J., Holst, J. J., Clark, P. M., Ellard, S., Søvik, O., Polak, M., Hattersley, A. T., and Neonatal Diabetes International Collaborative Group. (2006). Switching from insulin to oral sulfonylureas in patients with diabetes due to Kir6.2 mutations. N. Engl. J. Med. 355, 467-477.

Pitteloud, N., Quinton, R., Pearce, S., Raivio, T., Acierno, J., Dwyer, A., Plummer, L., Hughes, V., Seminara, S., Cheng, Y. Z., Li, W. P., Maccoll, G., Eliseenkova, A. V., Olsen, S. K., Ibrahimi, O. A., Hayes, F. J., Boepple, P., Hall, J. E., Bouloux, P., Mohammadi, M., and Crowley, W. (2007). Digenic mutations account for variable phenotypes in idiopathic hypogonadotropic hypogonadism. J. Clin. Invest. 117, 457-463.

Shariat, N., Ryther, R. C., Phillips, J. A. III, Robinson, I. C., and Patton, J. G. (2008). Rescue of pituitary function in a mouse model of isolated growth hormone deficiency type II by RNA interference. Endocrinology $149,580-586$

Received: 22 March 2011; accepted: 27 April 2011; published online: 25 May 2011.

Citation: Dattani MT and Martinez-Barbera J-P (2011) The future of genomic endocrinology. Front. Endocrin. 2:11. doi: 10.3389/fendo.2011.00011

This article was submitted to Frontiers in Genomic Endocrinology, a specialty of Frontiers in Endocrinology. Copyright (c) 2011 Dattani and Martinez-Barbera. This is an open-access article subject to a non-exclusive license between the authors and Frontiers Media SA, which permits use, distribution and reproduction in other forums, provided the original authors and source are credited and other Frontiers conditions are complied with. 